\title{
Experiencia del efecto de la docencia no presencial sobre la docencia inversa
}

Ana Vidaurrea,"*, M. Amparo Gámiz-González ${ }^{\mathrm{b}, \uparrow}$, Isabel Tort-Ausina ${ }^{\mathrm{c}, *}$, José Molina Mateo ${ }^{\mathrm{d}, *}$, María-Antonia Serrano ${ }^{\mathrm{e}, *}$, José M. Meseguer-Dueñas, ${ }^{\mathrm{f}, *}$, Jaime Riera ${ }^{\mathrm{g}, *}$, Rubén MartínCabezuelo $^{\mathrm{h}, *}$, Susana Quiles ${ }^{\mathrm{i}, *}$ y José A. Gómez-Tejedor ${ }^{\mathrm{j}, *}$

*Universitat Politècnica de València, Departamento de Física Aplicada. ${ }^{*}$ Universidad Internacionacional de Valencia, Área de Educación. *Universitat Politècnica de València, Departamento de Termodinámica Aplicada. ${ }^{a} v i d a u r r e @ f i s . u p v . e s, \quad{ }^{b}$ mariaamparo.gamiz@campusviu.es, ${ }^{c}$ isatort@fis.upv.es, djmmateo@fis.upv.es, 'emserranj@fis.upv.es, fjmmesegu@upv.es, g.jiera@fis.upv.es, hrubmarca@doctor.upv.es, isuquica@fis.upv.es, jjogomez@fis.upv.es

\section{\$EWWFW}

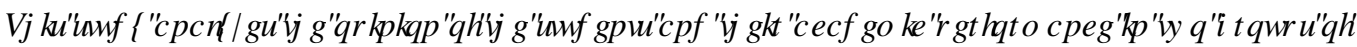
WKHIXENAFWRII3 KI UFVIRU\&RP SXWU6FIHQFHRIWKH\%

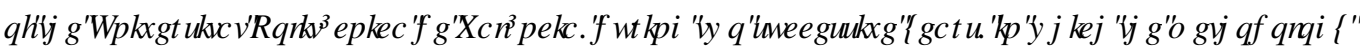

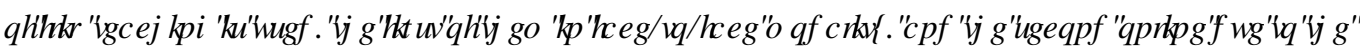



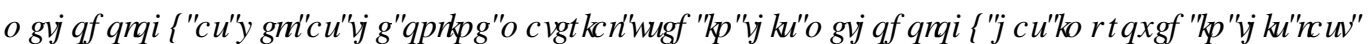



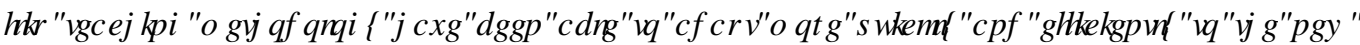



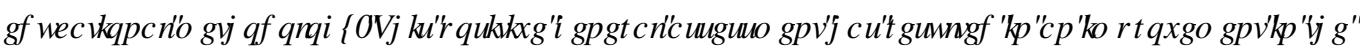



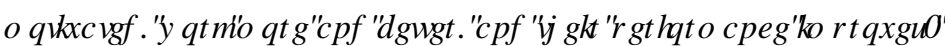

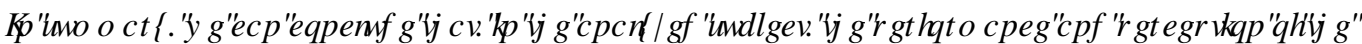



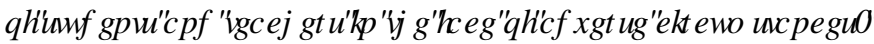

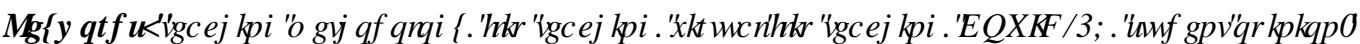

[

\section{HXP HQ}


DOP QRV GH QD DMJQDWWD ) XQGDP HQWRV ) t UFRV GH (DI LQRLP





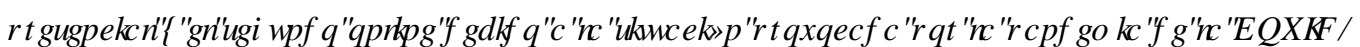

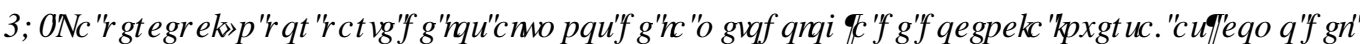

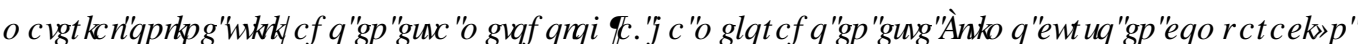

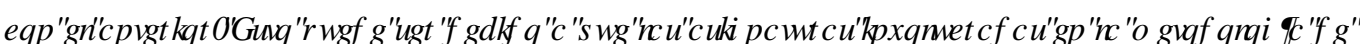



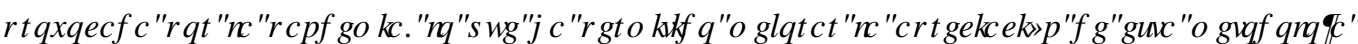

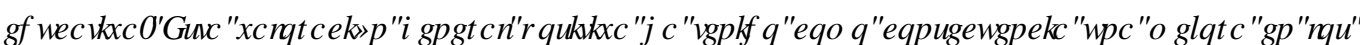






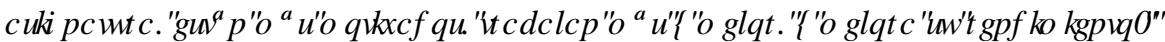

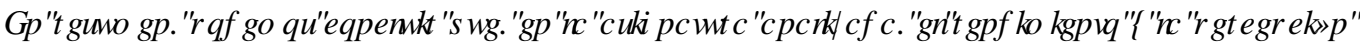

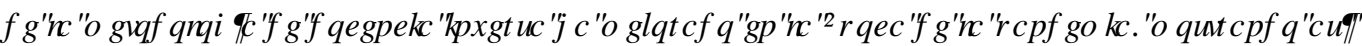

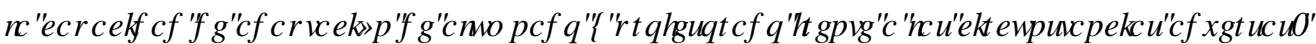

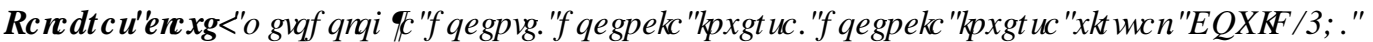 RSIQYYQHMKGDQMAT}

\section{Introducción}

Igual que en otros ámbitos de nuestras vidas, la pandemia del COVID-19 ha impactado de lleno en la docencia universitaria, obligando a incorporar la docencia RQQQH síncrona y asíncrona, como una herramienta de trabajo general en la docencia y el aprendizaje. Esta situación ha implicado que tanto la programación, como la organización y utilización de material docente por parte del profesorado, además de reflejar los contenidos claros y organizados de la materia, también deben incorporar cierto grado de motivación, anteriormente transmitido en el aula, para mantener un nivel de atención suficiente y conseguir que el alumnado no abandone el curso académico. Del mismo modo, los estudiantes se han visto obligados a adaptarse a la situación, la cual dependía en gran medida de sus condiciones (contar con un espacio de trabajo adecuado, con los medios tecnológicos idóneos, con un entorno familiar favorable, etc.), lo que ha repercutido en su formación y rendimiento académico.

Al cambiar de escenario, las metodologías tradicionales implementadas en el aula hasta ahora se han visto modificadas, ganando mayor notoriedad las metodologías activas como el caso de la Docencia Inversa (DIN). En las asignaturas en las que ya se estaba aplicando la metodología DIN se comenzó de un punto de partida más favorable que en aquellas en las que se seguían las metodologías tradicionales. Recordemos que en la metodología DIN se han modificado las condiciones de dónde y cuándo los estudiantes realizan sus tareas de aprendizaje. Mientras que en la metodología tradicional los conceptos nuevos son explicados por el docente en clase y el alumno luego estudia estos contenidos en casa, en la DIN los alumnos preparan el contenido de la clase antes de acudir al aula (viendo videos o leyendo textos), y la clase se dedica al trabajo y a la discusión en grupo sobre dichos contenidos, con la supervisión del profesor (Colomo-Magaña, 2020; Lindeiner-Stráský, 2020). El contar con gran cantidad de materiales diseñados para el trabajo autónomo del alumno: vídeos, exámenes de autoevaluación, tareas,...permitió concentrar la energía en las actividades de docencia RQDQH síncrona en las que se intentaba mantener el interés por la materia a través de actividades individuales y grupales. Ismail y Abdulla (Ismail, 2019) introducen el concepto de "Virtual Flipped Classroom" y muestran los beneficios de la conjunción de la docencia inversa y la docencia $R Q Q Q H$

Han sido muchos los trabajos realizados hasta el momento donde se ha analizado tanto la opinión de los estudiantes como el análisis de los resultados obtenidos tras la implementación de la DIN en comparación con la metodología tradicional (Gómez-Tejedor, 2020a; Gómez-Tejedor, 2020b; He, 2016)ПAlgunos de estos trabajos han resaltado las dificultades que esto implica en la docencia de primeros cursos (McCarthy, 2016; Tomas, 2019). En este trabajo, nos hemos centrado en el efecto que la adaptación a las condiciones impuestas por la pandemia, en concreto la docencia inversa virtual, ha tenido sobre los resultados, tanto a nivel de resultados académicos como de opinión de los estudiantes.

En este trabajo se analiza la experiencia llevada a cabo con dos grupos de alumnos de la asignatura Fundamentos Físicos de la informática (FFI), del primer cuatrimestre del primer curso del Grado en Ingeniería Informática (GII) de la Universitat Politècnica de València (UPV), durante dos cursos académicos, ambos siguiendo la metodología de docencia inversa, el primero de ellos presencial y el segundo RQQQHdebido a la situación de pandemia. Con el fin de comprender algunos de los resultados, 
éstos se comparan con los de alumnos del Grado en Ingeniería Electrónica y Automática Industrial (GIEAI) de la UPV.

\section{Descripción de la experiencia}

Durante el curso 2019-20 se inicia una experiencia de docencia inversa con alumnos del Grado en Ingeniería Informática (GII) de la Universitat Politècnica de València (UPV). De los nueve grupos de la asignatura Fundamentos Físicos de la informática (FFI), del primer cuatrimestre del primer curso, la experiencia se realiza en dos grupos de 50 alumnos cada uno, los que realizan la docencia en valenciano. Son una muestra representativa de los 450 alumnos, con una distribución de notas de PAU semejantes a la del total. Una característica del alumnado del GII es el porcentaje muy bajo de alumnas (en el curso 2019-20 el 5\% y en el 2020-21 el 8\%), que es más llamativa aún si se compara con el 30\% a que se llegó hace 20 años. Esto merecería algún estudio en clave de género.

La metodología utilizada está basada en tres pilares: el primero es la programación detallada del trabajo que los alumnos deben realizar antes, durante y después de las clases con el profesor. El segundo es que los alumnos disponen en todo momento de la totalidad del material de trabajo necesario para seguir la programación, alojado en una microweb de la asignatura. En la microweb, organizada de forma secuencial con la planificación del trabajo de los alumnos, se aloja material escrito, vídeos de contenidos teóricos, vídeos de contenidos prácticos, ejercicios de autoevaluación de corrección automática, y todo el material utilizado por el profesor en clase (presentaciones, hojas de cálculo, enlaces a contenidos externos...). El tercer pilar es el trabajo de tutoría, en la que el contacto entre profesor y alumno es bajo demanda, iniciándose mediante correo electrónico o chat. En la mayor parte de los casos los temas planteados se resuelven mediante el mismo canal de comunicación inicial, y en menor número de casos es necesaria una cita presencial, $\mathrm{y}$, actualmente, mediante videoconferencia.

La asignatura FFI tiene 6 créditos, de los que 4,5 son de aula y 1,5 de laboratorio. Los contenidos son de electricidad y física de semiconductores, y los objetivos de aprendizaje y las competencias que adquieren los alumnos, fijadas para la asignatura, la sitúan en un nivel de exigencia moderado dentro de las asignaturas de fundamentos físicos de primeros cursos en el resto de los grados en ingeniería impartidos en la UPV.

En el curso 2020-21 las características de los grupos y perfil de los alumnos no se ha modificado, y el profesorado encargado de la docencia y planificación de la asignatura se han mantenido. Debido a la situación provocada por la COVID-19 hay un cambio fundamental: todas las clases han sido "RQDQH" síncronas mediante la plataforma 0 IFLRVIWTHDP V("Microsoft Teams," 2020), que quedaban grabadas. La asistencia a la clase síncrona ha ido evolucionando desde un $90 \%$ al inicio del curso a un $60 \%$ hacia el final. Los visionados de las clases asíncronas han seguido una evolución en paralelo, pasando de más de 70 visionados al principio de curso a 30 hacia el final, cuando hay 98 alumnos matriculados en la asignatura (dos grupos).

Para conocer la opinión de los alumnos se elaboró una encuesta que fue contestada durante las dos últimas semanas de la asignatura por el $45 \%$ de los alumnos. La encuesta constaba de preguntas sobre la metodología utilizada, sobre diferentes actividades concretas empleadas, sobre su trabajo, y preguntas abiertas para que expresaran aspectos positivos, negativos y propuestas de mejora sobre la metodología de la asignatura. Una característica de la asignatura FFI es el bajo interés que despierta entre los alumnos, comparada con asignaturas similares en otras titulaciones de la UPV. En la tabla 1 se comparan los resultados de las preguntas sobre interés y utilidad de la asignatura comparados con los obtenidos en una encuesta paralela realizada a alumnos del Grado en Ingeniería Electrónica y Automática Industrial

(c)) BY-NC-ND 2021, Universitat Politècnica de València

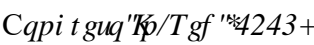


(GIEAI) de la UPV. De las tres preguntas posiblemente la clave esté en la tercera sobre la utilidad de los contenidos de la asignatura para otras asignaturas del grado: el promedio ponderado en el GII se valora con 4 puntos y en el GIEAI con más de 8 puntos. Además, en la figura 1 se puede observar la polarización en la opinión sobre la utilidad y sobre su uso en otras asignaturas, con un máximo relativo tanto en valoraciones positivas como negativas.

\begin{tabular}{|c|c|c|c|c|}
\hline & & GII & & GIEAI \\
\hline & $2019-20$ & $2020-21$ & Mejora & $2019-20$ \\
\hline $\begin{array}{c}\text { Estoy muy interesada/o en el contenido de esta } \\
\text { asignatura. }\end{array}$ & 5,5 & 6,3 & $18 \%$ & 7,7 \\
\hline $\begin{array}{c}\text { Después de este cuatrimestre, esta asignatura me } \\
\text { será muy útil. }\end{array}$ & 3,8 & 5 & $19 \%$ & 7,7 \\
\hline $\begin{array}{c}\text { Necesitaré los contenidos de esta asignatura en } \\
\text { otras posteriores. }\end{array}$ & 4 & 4,4 & $7 \%$ & 8,1 \\
\hline
\end{tabular}

En la tabla 1 y en la figura 1 se puede observar que los resultados han mejorado en el GII con la docencia RQDQHdel curso 2020-21 (sin llegar a aproximarse a los del GIEAI). Esto podría deberse a diferentes causas que van desde un mayor rodaje de la metodología utilizada en la asignatura en un contexto de poca simpatía hacia ella, hasta un clima de empatía y comprensión entre profesor y alumnos propiciado por las circunstancias (covid, vulnerabilidad, prueba continua de los límites de nuestras capacidades de trabajo, comunicación no verbal limitada, falta de realimentación directa...).

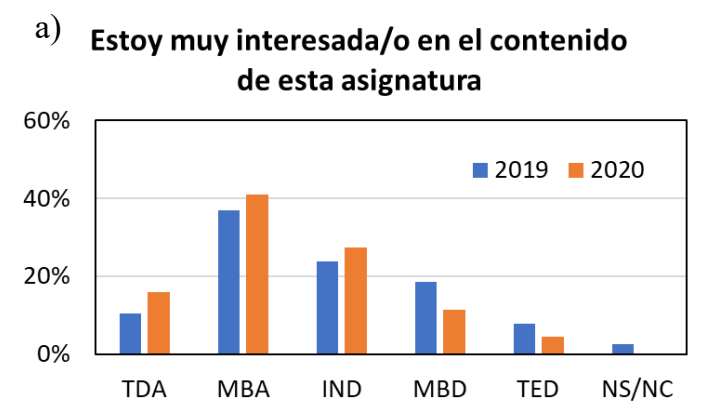

b) Después de este cuatrimestre, esta
asignatura me será muy útil.

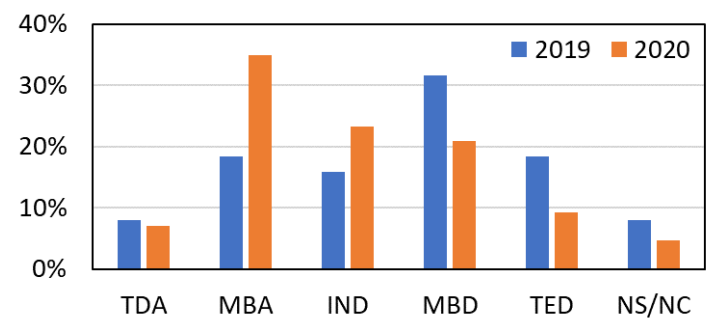

c) Necesitaré los contenidos de esta asignatura en otras posteriores.

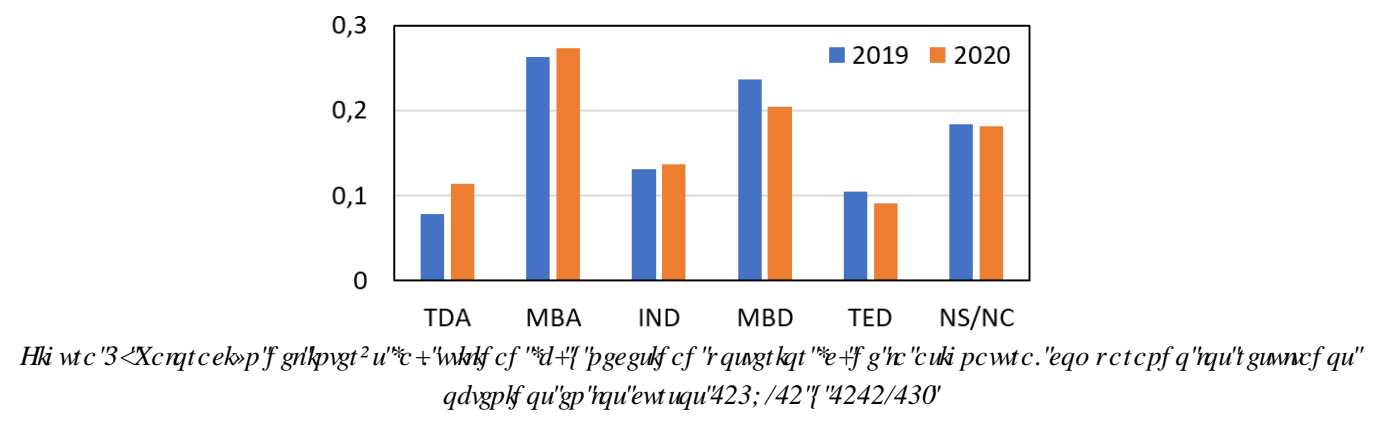




\section{Objetivos}

El objetivo de este trabajo es comparar la metodología de docencia inversa empleada en el Grado en Ingeniería Informática en dos años consecutivos, el segundo de ellos marcado por la pandemia de la COVID-19. Este análisis se realiza mediante la comparación de los resultados de una encuesta de opinión realizada en dos cursos consecutivos, así como mediante la comparación de las calificaciones académicas obtenidas por los alumnos en esos mismos años.

\section{Resultados}

\subsection{Valoración de la metodología empleada}

En nuestra experiencia de docencia inversa en primeros cursos realizadas en la UPV, la valoración del alumnado de la metodología empleada es baja (Gómez-Tejedor, 2020b). En el caso del GII, aunque hay una mejora en la valoración de la metodología en el segundo año respecto al primero, esta se encuentra bastante por debajo de la de los alumnos del GIEAI (tabla 2). No parece que la realización de las clases y tutorías por web haya modificado sustancialmente su opinión. No les gusta la metodología y no quieren que se extienda a otras asignaturas. Es una constante que se relaciona con el cambio en los hábitos de trabajo de los alumnos, acostumbrados en toda su formación previa a una metodología tradicional.

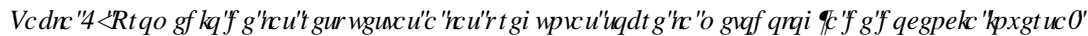

\begin{tabular}{c|ccc|c}
\hline & \multicolumn{3}{c}{ GII } & GIEAI \\
\hline $\begin{array}{c}\text { Prefiero este formato de docencia (estudiar antes } \\
\text { en casa los contenidos que se complementan en } \\
\text { clase) frente a un formato de clase tradicional. }\end{array}$ & 2,8 & 3,9 & $15 \%$ & $2019-20$ \\
\hline $\begin{array}{c}\text { Preferiría tener más asignaturas con este tipo de } \\
\text { formato (trabajar antes en casa y después en } \\
\text { clase). }\end{array}$ & 3 & 3,6 & $9 \%$ & 5,7 \\
\hline
\end{tabular}

Al pedir que valoren la asignatura y sus componentes presencial, en clase y por web, y contenidos en la microweb de la asignatura (tabla 3 ) se encuentra una mejora considerable entre los dos cursos académicos (hasta un 56\% de mejora en los contenidos RQQQH, y de poco más del $50 \%$ que la consideraban excelente o buena en el curso 2019-20, pasan al 90\% en el 2020-21. También mejora la valoración global de la asignatura, que es buena para un 65\% de los alumnos en el curso $2020-21$ frente a un 30\% en el curso 2019-20. En un cambio tan radical se debe tener en cuenta que, entre otros factores, en el curso 2020-21 toda la docencia se ha realizado RQDQH La valoración en el GII en el curso 2020-21 se sitúa en el mismo nivel que para los alumnos del GIEAI.

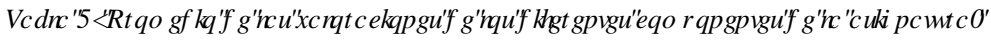

\begin{tabular}{c|ccc|c}
\hline \multicolumn{2}{c}{ GII } & \multicolumn{2}{c}{ GIEAI } \\
\hline Contenidos RQDQHde la docencia. & $2019-20$ & $2020-21$ & Mejora & $2019-20$ \\
\hline $\begin{array}{c}\text { Componente presencial de la docencia/ Clases } \\
\text { "RQQQH" con el profesor (parte síncrona de la } \\
\text { docencia). }\end{array}$ & 5,9 & 8,2 & $56 \%$ & 7,9 \\
\hline La asignatura en su conjunto & 4,9 & 7,1 & $43 \%$ & 7,9 \\
\hline
\end{tabular}




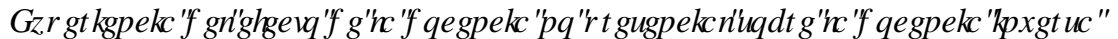

La docencia no presencial durante el curso 2020-21 ha supuesto tener que introducir nuevos elementos que compensen la falta de un contacto directo entre profesor y alumnado. En la tabla 4 y figura 2 se puede observar como la valoración de todos los elementos empleados es buena. Destacan los vídeos de ejercicios y teoría y las grabaciones de clase son consideradas excelentes o buenas en un porcentaje superior al $90 \%$. En el otro extremo se sitúan las prácticas de laboratorio, que han seguido una metodología tradicional pero realizadas RQQQHsíncronas mediante un simulador, de las que el $50 \%$ las ha considerado como excelentes o buenas. En la figura 3 se puede observar que la opinión sobre las prácticas virtuales está muy polarizada, presenta dos máximos, con porcentajes de alumnos parecidos a favor y en contra del modelo utilizado.

Tabla 4: Promedio de las valoraciones de diferentes elementos de la metodología empleada.

\begin{tabular}{|c|c|c|}
\hline & & GII \\
\hline \multirow{5}{*}{$\begin{array}{l}2019-21 \\
\text { docencia } \\
\text { inversa }\end{array}$} & & $2020-21$ \\
\hline & Documentos escritos de la asignatura por temas & 6,9 \\
\hline & Vídeos con teoría y resolución de problemas & 8,3 \\
\hline & Tarea inicial de síntesis de contenidos y resolución de dudas por el profesor & 7,9 \\
\hline & Presentaciones en PowerPoint & 6,6 \\
\hline \multirow{4}{*}{$\begin{array}{l}\text { Introducidos en } \\
2020-21 \\
\text { docencia } \\
\text { inversa }\end{array}$} & Propuesta de realización de "problemas semanales" & 7,4 \\
\hline & $\begin{array}{l}\text { Vídeos con la resolución de los "problemas semanales" (escritura sobre } \\
\text { cuaderno) }\end{array}$ & 8,8 \\
\hline & Grabaciones en "Stream" de las clases & 8,9 \\
\hline & "Kahoot" de cada tema (actividades de gamificación) & 7,8 \\
\hline $\begin{array}{l}2020-21 \text { no } \\
\text { docencia } \\
\text { inversa }\end{array}$ & Prácticas de laboratorio virtuales ("RQQQH ) & 5,1 \\
\hline
\end{tabular}








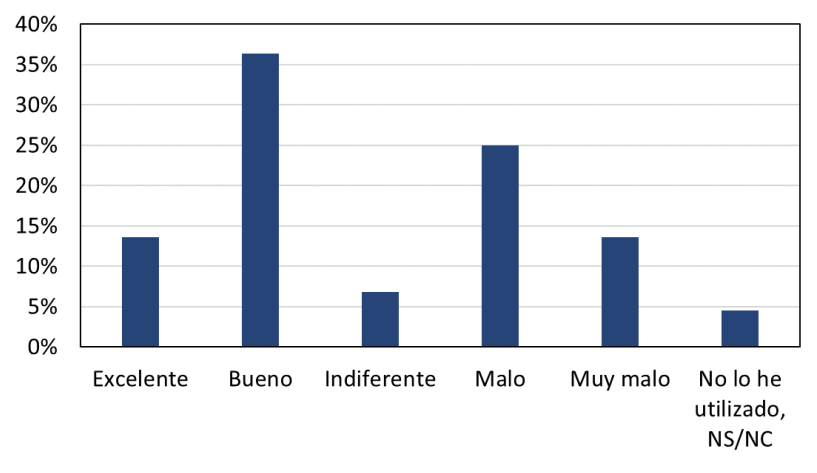

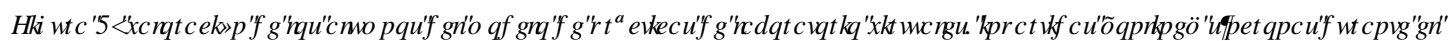



[

\subsection{Efecto de la experiencia sobre los resultados académicos}

En esta sección analizamos los resultados finales de los alumnos del GII en el curso 2020-21 comparándolos con los del curso previo a la pandemia provocada por la COVID-19 (figura 4). Lo primero que se constata es un incremento en los alumnos que han seguido el curso sin abandonar. Se ha pasado del 86\% en el curso 2019-20 (79 alumnos de 91) al 96\% en el 2020-21 (93 de 97). Hay un incremento significativo en el promedio de las notas, que coincide prácticamente con la mediana. La desviación estándar se mantiene en los dos cursos en el $17 \%$. La mejora en el promedio ( 0,7 puntos) supone un $17 \%$ sobre la mejora posible. La distribución de calificaciones sigue una forma de distribución normal en los dos cursos, con el máximo situado en los aprobados, entre 5 y 7 puntos (figura 4).

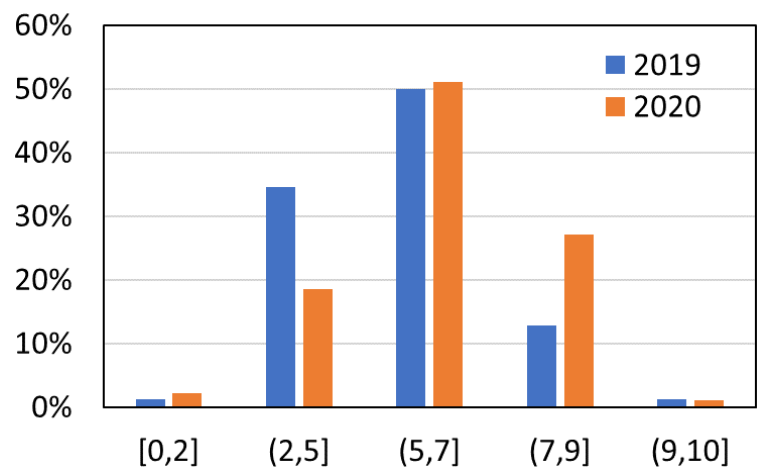

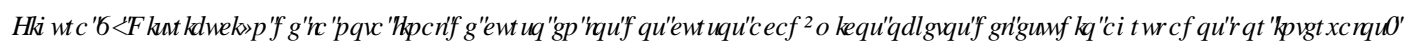

Para analizar el efecto de cómo la mejora ha afectado a los diferentes grupos de alumnos, clasificados por terciles en función de la nota final del curso, hemos dividido en tres tercios de igual número de alumnos en cada curso, siendo el primer tercil el de notas inferiores y el tercer tercil el de notas superiores (figura 5). 


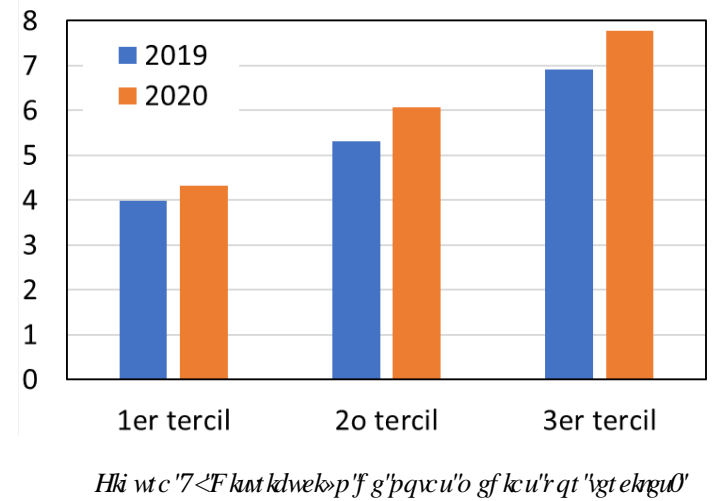

Podemos observar que el promedio de notas en los tres terciles mejora, es decir, la mejora en las calificaciones afecta a los tres grupos. Pero esa mejora es más importante en el tercero. Definiendo la mejora como la diferencia entre los promedios de notas en el curso $2020-21\left(N_{20 / 21}\right)$ y $2019-20\left(N_{19 / 20}\right)$ relativo a la diferencia entre el valor máximo, 10, y el promedio del curso 2019-20 (\%Rापिए),

$$
\text { Mejora }=\frac{N_{20 / 21}-N_{19 / 20}}{10-N_{19 / 20}}
$$

en la figura 6 podemos observar que la mejora ha afectado en mayor medida al tercer tercil, que se corresponde con los alumnos de mejor nota.

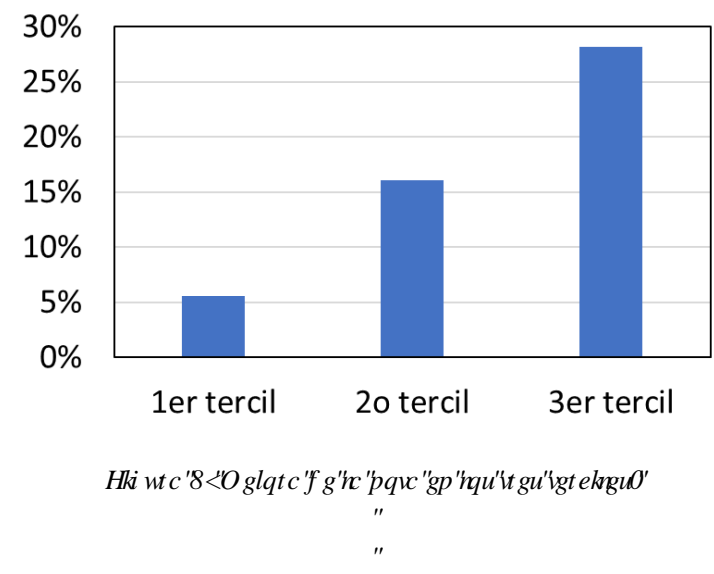

\subsection{Opinión de los estudiantes, resultados de las preguntas abiertas.}

En ambos cursos académicos se les pregunta a los estudiantes de GII que destaquen los aspectos positivos, negativos y propuestas de mejora de la asignatura. En el curso 2020-21 se añaden dos preguntas en las que se les pide que valoren la adaptación de los profesores, así como de ellos mismos, a la situación provocada por la pandemia.

Analizando las respuestas del alumnado se observa que hay un número alto de respuestas ( 33 el primer año y 35 el segundo). En relación a la metodología empleada en la asignatura, los estudiantes de ambos cursos, de forma bastante unánime valoran sobre todo positivamente la gamificación (KAHOOTS, https://kahoot.com/) y los problemas semanales realizados en forma de tutorías. También destacan los videos explicativos y la gran cantidad de material docente disponible que les ayuda a seguir las clases y llevar la asignatura al día. Otro punto importante que valoran es la posibilidad de visualizar los vídeos y las clases en cualquier otro momento, posibilidad que se ofrecía el primer año a través de los vídeos 
grabados por el profesor y a lo que se añadían la grabación de las clases en la docencia $R Q Q Q H$ Relacionado con la nueva situación debido a la COVID-19, valoran que el profesor se esfuerce en hacer el tema lo más interesante y agradable posible a pesar de los problemas de la enseñanza RQQQH

En relación a la metodología DIN hay opiniones diversas, habiendo estudiantes que se muestran abiertamente en desacuerdo. En concreto un estudiante indica: “...en mi opinión, explicar física con la docencia inversa es mala idea, se debería dejar más libertad al alumnado para hacer un ejercicio que él quiera sin tener que estar pendiente de tener que leerse un documento ni hacer los ejercicios mandados...", También piensan que la cantidad de tareas obligatorias es excesiva.

En cuanto a la labor de adaptación de la enseñanza realizada por los profesores, los estudiantes, en general, valoran muy positivamente el esfuerzo realizado durante la complicada situación que estamos viviendo. Consideran que han hecho un trabajo de adaptación muy bueno y que han mostrado interés y preocupación por el alumnado. Aunque no estén en general satisfechos con la docencia $R Q D Q H$ han valorado de forma excelente el trabajo realizado por el profesorado.

Respecto a la valoración que el alumnado hace de su trabajo, hay diversidad de opiniones. Hay estudiantes que piensan que se han esforzado y han hecho un buen trabajo en la asignatura, otros piensan que deberían de haberse esforzado más y que les ha faltado perseverancia, algunos achacan esto último a que no han sabido adaptarse a la docencia $R Q Q Q H$

\section{Conclusiones}

El análisis de los resultados, calificaciones y opinión, de estos dos cursos académicos consecutivos, nos permite comparar y obtener información acerca del efecto de la metodología DIN empleada en el Grado en Ingeniería Informática y, en concreto, sobre los cambios notables que se han generado debido a la docencia no presencial. A partir de los datos obtenidos en las encuestas de opinión se puede observar cómo, partiendo de un bajo nivel de motivación del alumnado, y a pesar de las dificultades de este pasado curso, el rendimiento y la motivación han mejorado. La percepción de la metodología de docencia inversa ha mejorado por parte de los alumnos durante este último curso en comparación con el anterior. Esto puede ser debido a que, en una situación social adversa, provocada por la pandemia, las asignaturas que impartían docencia utilizando la metodología DIN han podido adaptarse de forma más rápida y eficaz, lo que ha permitido apreciar la utilidad de esta metodología educativa. Esta situación también ha permitido que la opinión general acerca de los materiales RQQQHpor parte de los alumnos del Grado en Informática haya mejorado hasta situarse al nivel de los alumnos del Grado en Electrónica. Esta valoración general positiva ha tenido como consecuencia una mejora en los resultados académicos de los estudiantes. Cuando los alumnos valoran mejor la asignatura, están más motivados, trabajan más y mejor, y mejora su rendimiento, especialmente en los alumnos del tercir superior. Por último, si observamos las opiniones obtenidas acerca de las prácticas de laboratorio virtuales (no DIN), nos encontramos frente a una opinión muy polarizada, lo que indica que se debe trabajar para mejorarlas en cursos posteriores, y conseguir que la aceptación por parte de los alumnos sea generalizada.

En resumen, podemos concluir que, en la asignatura analizada, el rendimiento y la percepción de la metodología de docencia inversa ha mejorado en la época de la pandemia, mostrando así la capacidad de adaptación de alumnado y profesorado frente a las circunstancias adversas, y la situación de ventaja frente a situaciones sobrevenidas de la metodología DIN. 


\section{Agradecimientos}

Los autores agradecen al Instituto de Ciencias de la Educación de la Universitat Politècnica de València su apoyo al grupo de Innovación e-MACAFI y a los Proyecto PIME/2018/B25 y PIME/2018/B26.

\section{Referencias}

BAO, L. (2006). "Theoretical comparisons of average normalized gain calculations" HQ \$PHUFDQ - RXLQDORI3 KI UFV, vol. 74, issue 10, p. 917-922. https://doi.org/10.1119/1.2213632

COLOMO-MAGAÑA, E., SOTO-VARELA, R., RUIZ-PALMERO, J., y GÓMEZ-GARCÍA, M. (2020). "University students' perception of the usefulness of the flipped classroom methodology" HQ ( GXFDMRQ6FHQFH, vol. 10, issue 10, p. 1-19. https://doi.org/10.3390/educsci10100275

GÓMEZ-TEJEDOR, JOSÉ A., VIDAURRE, A., TORT-AUSINA, I., MOLINA-MATEO, J., SERRANO, M.-A., MESEGUER-DUEÑAS, J. M., MARTÍNEZ SALA, R..M. y RIERA, J. (2020a). "Effectiveness of flip teaching on engineering students' performance in the physics lab" HQ\&RP SXWUW ૧ ૧( GXFDMRQ vol. 144, p. 103708. https://doi.org/10.1016/j.compedu.2019.103708

GÓMEZ-TEJEDOR, JOSÉ ANTONIO, TORT- AUSINA, I., VIDAURRE, A., MESEGUER DUEÑAS, J. M., MOLINA - MATEO, J., y RIERA, J. (2020b). "Hacia una aplicación efectiva de Metodología Inversa en las asignaturas de Física de primeros cursos de Grado" en IN-RED 2020, p. 83-94. https://doi.org/10.4995/inred2020.2020.11934

HE, W., HOLTON, A., FARKAS, G., y WARSCHAUER, M. (2016). "The effects of flipped instruction on out-of-class study time, exam performance, and student perceptions" HQ/ HDQQQJ $D Q Q G$, QWWXFWRQ vol. 45, p. 61-71. https://doi.org/10.1016/j.learninstruc.2016.07.001

ISMAIL, S. S., y ABDULLA, S. A. (2019). "Virtual flipped classroom: New teaching model to grant the learners knowledge and motivation" HQ-RXLQDORID7HFQRRJ $\triangle D Q C F 6 F H Q F H($ GXFDURQ vol. 9, issue 2, p. 168-183. https://doi.org/10.3926/jotse.478

MCCARTHY, J. (2016). "Reflections on a flipped classroom in first year higher education" HQ, WXHVLQ ( GXFDMRQDO5 HMDFK, vol. 26, issue 2, p. 332-350.

"Microsoft Teams." (2020).

TOMAS, L., EVANS, N. (SNOWY), DOYLE, T., y SKAMP, K. (2019). "Are first year students ready for a flipped classroom? A case for a flipped learning continuum" $H Q$, QUHQDURQDO-RXLQDORID ( GXFDURQDO7HFKRQRJ। ILQ + IJKHU ( GXFDURQ vol. 16, issue 1. https://doi.org/10.1186/s41239-0190135-4 